\title{
Adaptive selection of antenna grouping and beamforming for MIMO systems
}

\author{
Kyungchul Kim, Kyungjun Ko and Jungwoo Lee
}

\begin{abstract}
Antenna grouping algorithms are hybrids of transmit beamforming and spatial multiplexing. With antenna grouping, we can achieve combining gain through transmit beamforming, and high spectral efficiency through spatial multiplexing. In an independent identically distributed channel, the antenna grouping method has better bit error rate (BER) performance than the beamforming method. However, if the channel is correlated, then the BER performance of antenna grouping degrades. In that case, it is better to use beamforming instead of antenna grouping. In this article, we investigate the mode selection algorithms which select between the antenna grouping and the beamforming modes. By selecting a suitable mode for a given channel, we can achieve more robustness of the system performance. We introduce several mode selection criteria as well as a low complexity criterion which is derived from a low complexity antenna grouping algorithm. Simulation results show that the proposed mode selection algorithm performs better than the antenna grouping and the beamforming modes in various channel conditions.
\end{abstract}

\section{Introduction}

Multiple-input and multiple-output (MIMO) systems have been investigated extensively for their high spectral efficiency and reliable transmission of data $[1,2]$ over single-input and single-output (SISO) systems. Through multiple transmit antennas, we can transmit several independent data streams by spatial multiplexing mode. We can also send only one data stream by transmit beamforming or diversity modes. With spatial multiplexing, we can achieve high spectral efficiency, but the reliability of data transmission gets worse especially when there is a correlation between antennas. On the other hand, we can obtain combining gain (SNR gain) by sacrificing spectral efficiency in the beamforming mode. ${ }^{\mathrm{a}}$

We assume an MIMO system which has $N_{t}$ transmit antennas and $N_{r}$ receive antennas. The availability of channel state information (CSI) at the transmitter helps to make the system more efficient [3]. Beamforming is one of the strategies which use the CSI at the transmit side. By singular value decomposition (SVD), it divides MIMO channel into $\min \left(N_{t}, N_{r}\right)$ SISO channels and transmits one data stream through the best SISO channel. It increases the received signal-to-noise ratio (SNR),

\footnotetext{
* Correspondence: junglee@snu.ac.kr

School of Electrical Engineering and Computer Sciences, Seoul National
} University, Seoul 151-744, Korea and improves the reliability. Especially in a highly correlated channel, beamforming is the best transmit strategy for the bit error rate (BER) performance. But transmission of only one stream can make beamforming inefficient with respect to spectral efficiency. When the bit per channel use (BPCU) is fixed, the modulation order of beamforming tends to be higher than that of spatial multiplexing, and the BER performance will be degraded in an independent identically distributed (IID) channel. The eigenmode transmission also uses SVD to find precoding matrix. In the eigenmode transmission, $\min \left(N_{t}, N_{r}\right)$ streams can be transmitted with adequate power allocation. To maximize the capacity, water-filling-based power allocation is optimal, while inverse water-filling minimizes the mean square error [4]. General multi-mode precoding [5-7] can also be used, and it adapts the number of transmission streams to minimize the BER or maximize the capacity. In multi-mode precoding systems, each instantaneous channel prefers a particular mode.

Antenna grouping is a combination of beamforming and spatial multiplexing [8]. We also introduced some antenna grouping criteria [9]. When $N_{t}$ is larger than the $N_{r}, N_{t}$ transmit antennas can be partitioned into $N_{r}$ groups. The antennas in each group are used for beamforming, and an independent data stream is transmitted in each group. In short, antenna grouping transmits $N_{r}$ independent data

\section{Springer}


streams through partitioned beamforming. In this algorithm, we can improve the BER performance by achieving combining gain through beamforming, and multiplexing gain through spatial multiplexing. We assume that SVD is performed at the receiver instead of the transmitter, so that we need to feedback beamforming vector(s) or right unitary matrix of SVD instead of full CSI. Feedback information in antenna grouping is a beamforming vector $\left(N_{t} \times 1\right.$ vector) plus additional antenna grouping information while required feedback information in eigenmode transmission is an $N_{t} \times N_{r}$ matrix. As the antenna correlation increases, the BER performance of antenna grouping gets worse, and beamforming is the best strategy as mentioned earlier. In case of an ill-conditioned channel (i.e., the condition number of a matrix is large), the BER performance of antenna grouping may not be better than that of beamforming because we cannot send $N_{r}$ streams through an ill-conditioned channel. In average, an ill-conditioned channel occurs more frequently in a correlated channel. This is why beamforming is the best strategy in a highly correlated channel.

To overcome performance degradation in an ill-conditioned or a correlated channel, we propose to use mode selection for each instantaneous channel. We only consider beamforming and antenna grouping as the two possible modes in the mode selection algorithm to limit the feedback information and the complexity. Eigenmode transmission requires an $N_{t} \times N_{r}$ matrix feedback which is much more than beamforming and antenna grouping. Multi-mode precoding systems are complex because it considers all possible numbers of data transmission streams. The rest of this article is organized as follows. In Section 2, we provide the system model. We review the antenna grouping algorithms and introduce mode selection criteria in Sections 3 and 4. The proposed antenna grouping algorithm and the mode selection criterion are presented in Sections 3.5 and 4.5, respectively. Section 5 provides simulation results, and conclusions are given in Section 6.

\section{System model}

We assume that the receiver and the transmitter know the CSI. We also assume that the number of the transmit antennas is larger than that of receiver's $\left(N_{t}>N_{r}\right) . \mathbf{H}$ is a $N_{r} \times N_{t}$ matrix where $h_{i, j}$ is the path gain from the $j$ th transmit antenna to the $i$ th receive antenna. We assume a general correlated matrix channel so that $h_{i, j}$ and $h_{k, l}$ $(i \neq k$ or $j \neq l)$ may be correlated.

$$
\mathbf{H}=\mathbf{R}^{\frac{1}{2}} \mathbf{H}_{\mathbf{w}} \mathbf{T}^{\frac{1}{2}}
$$

where $\mathbf{R}$ and $\mathbf{T}$ are receiver and transmitter antenna correlation matrix, respectively. $h_{w_{i, j}}$, the $(i, j) t h$ element of $\mathbf{H}_{\mathbf{w}}$, is modeled as an independent and identical complex Gaussian distribution with 0 mean and unit variance. When the channel has IID Rayleigh fading, $\mathbf{R}$ and $\mathbf{T}$ in (1) is an identity matrix $\mathbf{I}$. The noise $\mathbf{n}$ is an AWGN vector with variance of $\sigma_{n}^{2}$ 's.

At first, in the antenna grouping mode, we partition $N_{t}$ transmit antennas into $N_{r}$ groups. The channel matrix $H$ is written as

$$
\mathbf{H}=\left[\begin{array}{lll}
\mathbf{h}_{1} & \mathbf{h}_{2} & \cdots \mathbf{h}_{N_{t}}
\end{array}\right]
$$

We partition the integer set from 1 to $N_{t}$ into $N_{r}$ groups, and name them as

$$
S_{1}, S_{2}, \ldots, S_{N_{r}} \text {. }
$$

Let $\left|S_{i}\right|=n_{i}$ (where $\left|S_{i}\right|$ is the cardinality of the set $S_{i}$ for $i=1, \ldots, N_{r}$ ), which satisfies

$$
n_{1}+\cdots+n_{N_{r}}=N_{t} .
$$

We can define a $N_{r} \times n_{i}$ sub-channel matrix $\mathbf{H}_{\mathbf{i}}$ 's as

$$
\mathbf{H}_{\mathbf{i}}=\left[\mathbf{h}_{s_{i 1}}, \ldots, \mathbf{h}_{s_{i_{i}}}\right]
$$

where $s_{i j}$ is the $j$ th element of the set $S_{i}$. We can obtain the beamforming vector of each sub-channel $\mathbf{w}_{\mathbf{i}}$ as the right singular vector corresponding to the largest singular value of the SVD of $\mathbf{H}_{\mathbf{i}}$. The received signal can be modeled as

$$
\mathbf{y}=\mathbf{H} \cdot \mathrm{W}_{\mathrm{AG}} \cdot \mathrm{x}_{\mathrm{AG}}+\mathbf{n}=\mathrm{H}_{\mathrm{AG}} \cdot \mathrm{x}_{\mathrm{AG}}+\mathbf{n}
$$

where $\mathbf{H}_{\mathrm{AG}}$ is effective channel of antenna grouping, $\mathbf{x}_{\mathrm{AG}}$ is the $N_{r} \times 1$ transmitted signal vector, $\left\|\mathbf{x}_{\mathbf{A G}}\right\|=$ 1. We assume equal power allocation so $\left|x_{\mathrm{AG}, i}\right|^{2}\left(\forall_{i} \leq\right.$ $N_{r}$ ) is always $\frac{1}{N_{r}}$ where $x_{\mathrm{AG}, i}$ is the $i$ th element of $\mathbf{x}_{\mathrm{AG}}$. $\mathbf{W}_{\text {AG }}$ is the $N_{t} \times N_{r}$ matrix and the $m$ th element of $\mathbf{w}_{n}$ corresponds to $\left[\mathrm{W}_{\mathrm{AG}}\right]_{s_{n m} n}$, and the other elements of the $n$th column of $\mathbf{W}_{\mathbf{A G}}$ are 0 ( $\left\|\mathbf{w}_{n}\right\|$ is normalized to 1$)$. For example, suppose that $\mathbf{W}_{\mathbf{A G}}$ is a $4 \times 2$ matrix, $\mathbf{w}_{1}=$ $(a, b)^{T}$, and $\mathbf{w}_{2}=(c, d)^{T}$. Note that $\left\|\mathbf{w}_{1}\right\|^{2}=a^{2}+b^{2}=$ 1 and $\left\|\mathbf{w}_{2}\right\|^{2}=c^{2}+d^{2}=1$. If the grouping is given by $S_{1}=\{1,4\}$ and $S_{2}=\{2,3\}$, then we then have

$$
\mathbf{W}_{\mathrm{AG}}=\left[\begin{array}{ll}
a & 0 \\
0 & c \\
0 & d \\
b & 0
\end{array}\right] .
$$

In the beamforming mode, we use the right singular vector corresponding to the maximum singular value of a given channel matrix. The received signal can be modeled as

$$
\mathbf{y}=\mathbf{H} \cdot \mathbf{w}_{\mathbf{B}} \cdot x_{B}+\mathbf{n}=\mathbf{h}_{\mathbf{B}} \times x_{\mathbf{B}}+\mathbf{n}
$$

The only difference between (5) and (7) is dimension of matrices. The dimensions of $\mathbf{W}_{\mathbf{A G}}$ and $\mathbf{x}_{\mathbf{A G}}$ are $N_{t} \times N_{r}$ 
and $N_{r} \times 1$, respectively. But the dimension of $\mathbf{w}_{\mathbf{B}}$ is $N_{t} \times 1$, and $x_{B}$ is a scalar. The $\mathbf{H}_{\mathrm{AG}}$ is $N_{r} \times N_{r}$ matrix, whereas $\mathbf{h}_{\mathbf{B}}$ is $N_{r} \times 1$ vector.

\section{Reviews of antenna grouping}

There are several antenna grouping techniques, which were introduced in [10].

\section{Sum capacity of sub-channels (Algorithm A1)}

In this algorithm, the grouping criterion is the sum capacity of sub-channels [8]. The sum capacity of subchannels is

$$
C_{S_{1}, S_{2}, \ldots, S_{N_{r}}}^{w_{1}, w_{2}, \ldots, w_{w_{r}}} \cong \log \left(1+\frac{\mathrm{SNR}}{N_{t}} \sum_{i=1}^{N_{r}} \mathbf{w}_{\mathbf{i}}^{H} \mathbf{H}_{\mathbf{i}}^{H} \mathbf{H}_{\mathbf{i}} \mathbf{w}_{\mathbf{i}}\right)
$$

Note that (8) is an approximation, and this algorithm is not optimal even in terms of capacity. To maximize (8), we need to search the sub-channel group that maximizes

$$
\sum_{i=1}^{N_{r}} \mathbf{w}_{\mathbf{i}}^{H} \mathbf{H}_{\mathbf{i}}^{H} \mathbf{H}_{\mathbf{i}} \mathbf{w}_{\mathbf{i}}
$$

\section{Minimum euclidean distance of received constellations (Algorithm A2)}

The minimum Euclidean distance of receive constellation is shown [10] as

$$
d_{\min }^{2}:=\min _{x_{i}, x_{j} \in X, x_{i} \neq x_{j}} \frac{\left\|\mathbf{H}_{\mathrm{AG}}\left(\mathbf{x}_{\mathbf{i}}-\mathbf{x}_{\mathbf{j}}\right)\right\|^{2}}{N_{r}}
$$

where $X$ is the set of all possible transmitted vector $\mathbf{x}_{\mathrm{AG}}$. We consider all possible effective channel $\mathbf{H}_{\mathbf{A G}}$ 's in (5). We calculate the minimum Euclidean distance of receive constellation for every possible $\mathbf{H}_{\mathbf{A G}}$, and find the optimal sub-channel $\mathbf{H}_{\mathbf{i}}$ 's and the optimal $\mathbf{W}_{\mathbf{A G}}$ that maximize (10).

\section{Minimum singular value of effective channel (Algorithm A3)}

A MIMO channel can be decomposed into multiple SISO channels by SVD, and the received SNR is proportional to the squared singular value of a channel. The BER performance is thus dominated by the minimum singular value. We find the minimum singular value of each $\mathbf{H}_{\mathbf{A G}}$, and pick the best $\mathbf{H}_{\mathbf{i}}$ 's and $\mathbf{W}_{\mathbf{A G}}$ which maximize the minimum singular value of $\mathbf{H}_{\mathbf{A G}}$.

\section{Effective channel capacity (Algorithm A4)}

Unlike Algorithm A1, this does not consider the sum capacity of sub-channels but overall channel capacity itself. As in other algorithms, for every possible effective channel $\mathbf{H}_{\mathbf{A G}}$, we calculate channel capacity

$$
C=\log \operatorname{det}\left(\mathbf{I}_{\mathbf{N}_{\mathbf{r}}}+\frac{1}{N_{r} \sigma_{n}^{2}} \mathbf{H}_{\mathrm{AG}}{ }^{H} \cdot \mathbf{H}_{\mathrm{AG}}\right) .
$$

We can then select the grouping and the precoding matrix which maximize (11).

\section{Based on normalized instantaneous channel correlation matrix (Algorithm A5)}

Transmit antennas which are highly correlated are grouped together and transmit antennas which are less correlated are separately grouped in this algorithm. Let us define a normalized instantaneous channel correlation matrix (NICCM) as

$$
\begin{gathered}
{[\mathbf{R}]_{i j}=\frac{1}{\left\|\mathbf{h}_{i}\right\| \cdot\left\|\mathbf{h}_{j}\right\|} \cdot\left[\mathbf{H}^{H} \mathbf{H}\right]_{i j}} \\
\mathbf{R}=\left[\begin{array}{cccc}
1 & r_{12} & \cdots & r_{1 N_{t}} \\
r_{12}^{*} & 1 & \cdots & r_{2 N_{t}} \\
\vdots & \vdots & \ddots & \vdots \\
r_{1 N_{t}}^{*} & r_{2 N_{t}}^{*} & \cdots & 1
\end{array}\right]
\end{gathered}
$$

In (13), if the amplitude of $r_{13}$ is large, then it means that the first and the third columns of $\mathbf{H}$ are more correlated than the other pairs. This can be interpreted as the correlation between the transmit antennas 1 and 3 is large.

Using this concept, we can devise a simple antenna grouping algorithm. For simplicity's sake, assume $N_{t}$ is 4 and $N_{r}$ is 2 . In a $4 \times 2$ system, $\mathbf{R}_{4 \times 2}$ is written as

$$
\mathbf{R}_{4 \times 2}=\left[\begin{array}{cccc}
1 & A & B & C \\
A^{*} & 1 & D & E \\
B^{*} & D^{*} & 1 & F \\
C^{*} & E^{*} & F^{*} & 1
\end{array}\right]
$$

For simplicity, we consider only the antenna grouping where the size of each group is 2 , which we call $(2,2)$ grouping. The possible antenna grouping cases are $(1,2 / / 3,4),(1,3 / / 2,4)$ and $(1,4 / / 2,3)$. We then compare $(\mid$ $A|+| F \mid),(|B|+|E|)$, and $(|C|+|D|)$. If $(|A|+|F|)$ is the largest, it means that the correlation between transmit antennas 1 and 2 and between transmit antennas 3 and 4 is larger than the others so we group $(1,2)$ and $(3,4)$ together which are denoted by $(1,2 / / 3,4)$. Similarly, if (| $B|+| E \mid)$ is the largest, then we use the grouping of $(1,3 / /$ $2,4)$. If $(|C|+|D|)$ is the maximum, then we use the grouping of $(1,4 / / 2,3)$. The advantage of this algorithm is that it reduces the search complexity significantly. This antenna grouping algorithm can be extended to any MIMO system where $N_{t}$ is an integer multiple of $N_{r}$. The BER performance of this algorithm is compared to other criteria in [9], and it is very close to others. 


\section{Mode selection}

As we compared each possible group with a certain criterion in antenna grouping, we can compare two modes (antenna grouping and beamforming) with a similar criterion. In this section, we provide several mode selection criteria similar to those of antenna grouping.

\section{Minimum Euclidean distance of received constellations (Algorithm M1)}

As (10), the minimum Euclidean distance of received beamforming constellation is

$$
d_{\min , B}^{2}:=\min _{s_{i}, s_{j} \in S_{,} s_{i} \neq s_{j}}\left\|\mathbf{h}_{\mathbf{B}}\left(s_{i}-s_{j}\right)\right\|^{2}=\lambda_{\max }^{2}(\mathbf{H}) \cdot d_{\min , b}^{2}
$$

where $S$ is the set of all possible transmitted signals $x_{B}, \lambda_{\max }(H)$ is the maximum singular value of $\mathbf{H}$, and $d_{\min , b}$ is the minimum Euclidean distance of the transmit beamforming constellation. The second equality is because $s_{i}$ 's are scalars in beamforming. If (10) is larger than (15), then we select the antenna grouping mode, and vice versa.

\section{Range of minimum distance (Algorithm M2)}

When the calculation of (10) is difficult, this approximated criterion can be used. In [11], they derived the range of received minimum constellation distance in received constellation. The minimum Euclidean distance of the received antenna grouping constellation is

$$
\lambda_{\min }^{2}\left(\mathbf{H}_{\mathbf{A G}}\right) \cdot \frac{d_{\min , a g}^{2}}{N_{r}} \leq d_{\min , a g}^{2} \leq \lambda_{\max }^{2}\left(\mathbf{H}_{\mathbf{A G}}\right) \cdot \frac{d_{\min , a g}^{2}}{N_{r}}
$$

where $\lambda_{\min }\left(\mathbf{H}_{\mathbf{A G}}\right), \lambda_{\max }\left(\mathbf{H}_{\mathbf{A G}}\right)$ are minimum and maximum singular values of $\mathbf{H}_{\mathbf{A G}} \cdot d_{\mathrm{min}, a g}$ is the minimum Euclidean distance of the transmit constellation in antenna grouping. In (15), we can easily calculate the minimum Euclidean distance of received beamforming constellation. As in [11], we compare $\lambda_{\min }^{2}\left(\mathbf{H}_{\mathrm{AG}}\right) \cdot \frac{d_{\min , a_{8}}^{2}}{N_{\mathrm{r}}}$ and $\lambda_{\max }^{2}(\mathbf{H}) \cdot d_{\min , b}^{2}$. If the former is larger than the latter, we select the antenna grouping mode, and vice versa.

\section{Effective channel capacity (Algorithm M3)}

The channel capacity of the two modes is

$$
\begin{aligned}
& C_{\mathrm{AG}}=\log \operatorname{det}\left(\mathbf{I}_{\mathbf{N}_{\mathbf{r}}}+\frac{1}{N_{r} \sigma_{n}^{2}} \mathbf{H}_{\mathrm{AG}}{ }^{H} \cdot \mathbf{H}_{\mathrm{AG}}\right) . \\
& C_{\mathrm{B}}=\log \left(1+\frac{1}{\sigma_{n}^{2}} \mathbf{h}_{\mathrm{B}}{ }^{H} \cdot \mathbf{h}_{\mathrm{B}}\right) .
\end{aligned}
$$

If $C_{A G}$ is larger than $C_{B}$, then we select the antenna grouping mode, and vice versa.

\section{Condition number of channel matrix (Algorithm M4)}

In the M2 algorithm, we compare $\lambda_{\text {min }}^{2}\left(\mathbf{H}_{\mathrm{AG}}\right) \cdot \frac{d_{\min , a_{s}}^{2}}{N_{r}}$ and $\lambda_{\max }^{2}(\mathrm{H}) \cdot d_{\min , b}^{2}$. According to the properties of a singular value, $\lambda_{\text {max }}\left(\mathbf{H} \cdot \mathbf{W}_{\mathbf{A G}}\right) \leq \lambda_{\max }(\mathbf{H}) \cdot \lambda_{\max }\left(\mathbf{W}_{\mathbf{A G}}\right) \cdot \lambda_{\max }$ $\left(\mathbf{W}_{\mathbf{A G}}\right)$ is 1 because columns of $\mathbf{W}_{\mathbf{A G}}$ is orthonormal, $\lambda_{\max }\left(\mathbf{H}_{\mathbf{A G}}\right) \leq \lambda_{\max }(\mathbf{H})$. As can be observed from Figure 1 which shows numerical results, $\lambda_{\max }\left(\mathbf{H}_{\mathrm{AG}}\right) \cong \lambda_{\max }(\mathbf{H})$. We can then simplify algorithm M2 into the comparison of $\lambda_{\max }\left(\mathbf{H}_{\mathbf{A G}}\right) / \lambda_{\text {min }}\left(\mathbf{H}_{\mathbf{A G}}\right)$ (condition number of $\mathbf{H}_{\mathbf{A G}}$ ) and $\sqrt{\frac{d_{\min , a_{g}}^{2}}{N_{r} \cdot d_{\min , b}^{2}}}$. If the former is larger than the latter, then we select the beamforming mode, and vice versa. In this mode selection algorithm, if the condition number of $\mathbf{H}_{\mathbf{A G}}$ is larger than the threshold, then the beamforming mode will be selected. It is expected that the beamforming mode will be selected in an ill-conditioned channel, then the antenna grouping mode will be selected in a well-conditioned channel.

\section{Based on NICCM (Algorithm M5)}

Conceptually, in the A5 algorithm, if the maximum of $(|A|$ $+|F|),(|B|+|E|)$, and $(|C|+|D|)$ is large, then it is safe to select antenna grouping. However, if the sum of smaller two of $(|A|+|F|),(|B|+|E|)$, and $(|C|+|D|)$ is also large, then it means that all columns of the channel matrix are close to each other, and the channel is ill conditioned, and the performance of antenna grouping degrades. When the sum of smaller two of $(|A|+|F|),(|B|+|E|)$, and $(|C|+\mid$ $D \mid)$ is large, then it is better to select the beamforming mode. Another important issue is how to determine the threshold value. But, as can be seen from Figure 2, this value is correlated with the condition number. By combining with the M4 algorithm, we can set an approximate threshold. If the off-diagonal sum of NICCM is larger than the threshold, then the beamforming mode is selected, and vice versa.

\section{Simulation results}

As for the correlation matrix of (1), we use the correlation matrix of channel from the TGn model of the IEEE $802.11 \mathrm{n}$ standard. We consider only the correlation of transmit antennas, and it may be a reasonable assumption in which mobile is surrounded with lots of scatterers. In simulation results, we add the performance of the eigenmode transmission with inverse water-filling as a reference, a $4 \times 2$ MIMO system is assumed. Figure 3 shows the performance of the algorithm M1 combined with the algorithm A2 when the BPCU is 4 for an IID channel. We transmit two data streams of QPSK for the antenna grouping and the eigenmode transmission methods, and one data stream of 16-QAM for the beamforming mode. The proposed mode selection performs better 


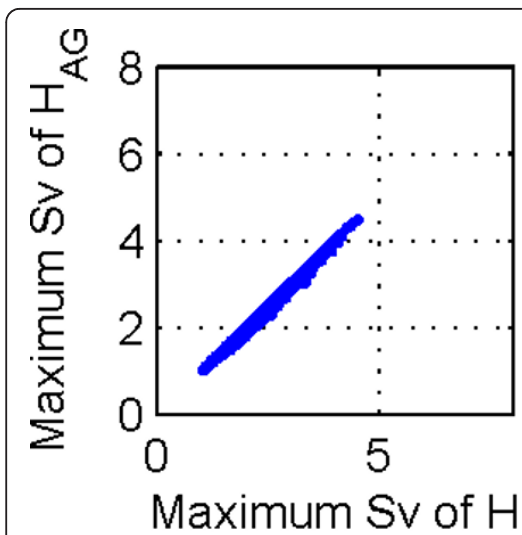

a

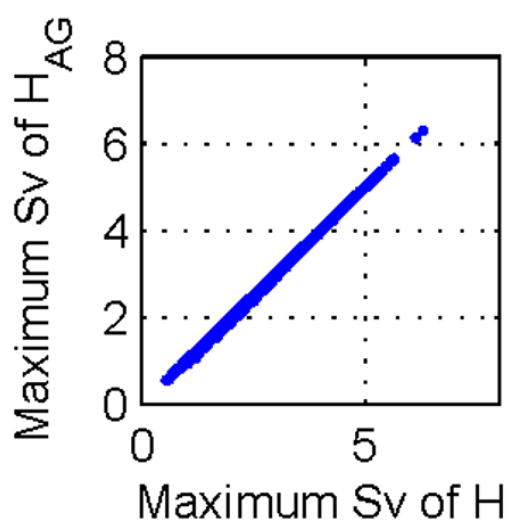

b

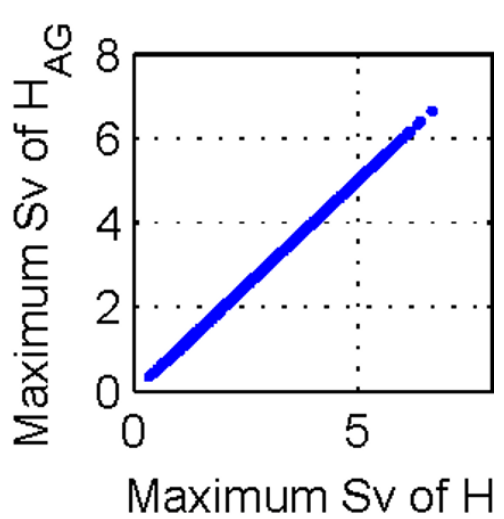

c

Figure 1 Relationship of $\lambda_{\max }(H)$ and $\lambda_{\max }\left(H_{A G}\right)$ : (a) IID channel, (b) correlated channel (AOD: $45^{\circ}, A S: 15^{\circ}$ ), and (c) correlated channel (AOD: $45^{\circ}$, AS: $6^{\circ}$ ).

than antenna grouping, beamforming, and eigenmode transmission in an IID channel. As can be observed from Figure 4, the proposed mode selection also performs better than the others in a correlated channel although the relative performance among beamforming, antenna, grouping and eigenmode transmission may change. As shown in [9], algorithm A2 is the best criterion for antenna grouping. But it is complex to use in practical systems especially with higher-order modulation.

Figure 4 shows the BER performance of mode selection algorithms with the A2 antenna grouping algorithm in highly correlated channels where the angle of departure (AOD) is $45^{\circ}$, and the angular spread (AS) is $6^{\circ}$. In this simulation, the BPCU is 8 . We use 16-QAM in the antenna grouping and the eigenmode transmission methods, and 256-QAM in the beamforming mode. In the high SNR region, the $\mathrm{M} 1$ algorithm has the best BER performance, and the others have similar performance. The proposed algorithm (M5) has performance similar to the M2 and the M4 algorithms while its complexity is lower.
In Figure 5, we also simulated mode selection algorithms with the A5 antenna grouping algorithm with the same condition. The A5 algorithm has slightly worse performance than the A2 algorithm. The overall performance of Figure 5 is slightly worse than the performance of Figure 4. However, the relative performance of the compared algorithms is similar in the two figures. The performance of the mode selection method is better than the antenna grouping, the beamforming, and the eigenmode transmission methods. As we mentioned earlier, the A5 antenna grouping algorithm has low complexity, and the M5 mode selection algorithm requires no additional calculation. The combination of the M5 and A5 algorithms also has low complexity.

In Figure 6, we use the combined algorithm of A5 and M5. The solid lines are for an IID channel and the dashed lines are for a highly correlated channel. As in Figures 4 and 5 , the performance of mode selection is the best. By examining Figure 2 and the condition number of the M4 algorithm, we can roughly obtain the desirable
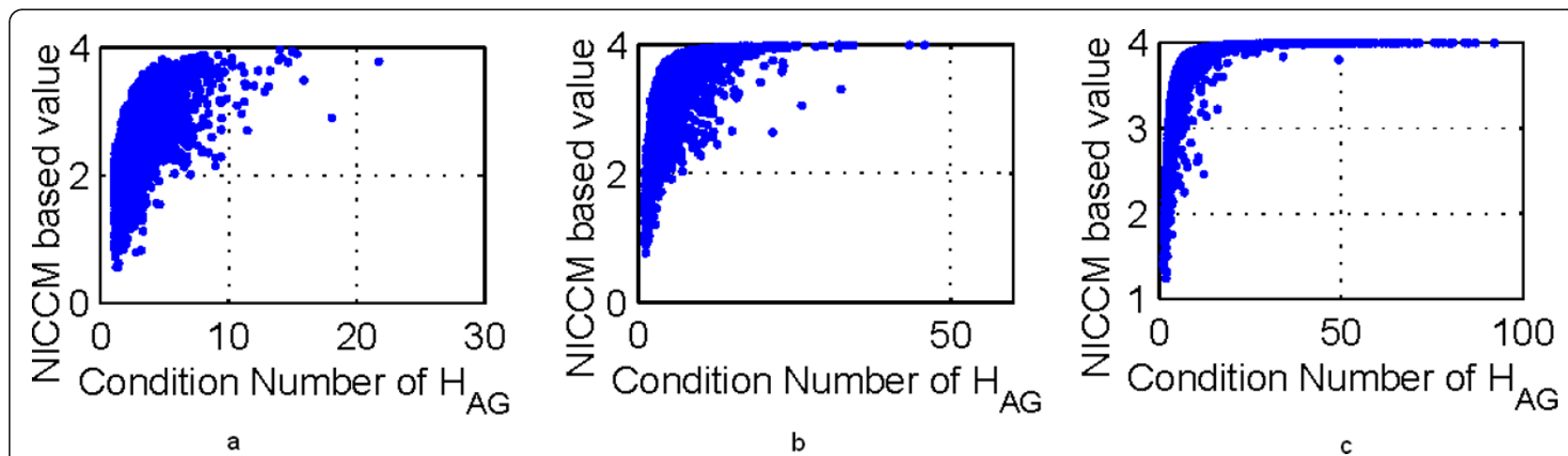

Figure 2 Relationship of the condition number and the NICCM value: (a) IID channel, (b) correlated channel (AOD: $45^{\circ}$, AS: $\left.15^{\circ}\right)$, and $(\mathbf{c})$ correlated channel (AOD: $45^{\circ}, \mathrm{AS}: 6^{\circ}$ ). 


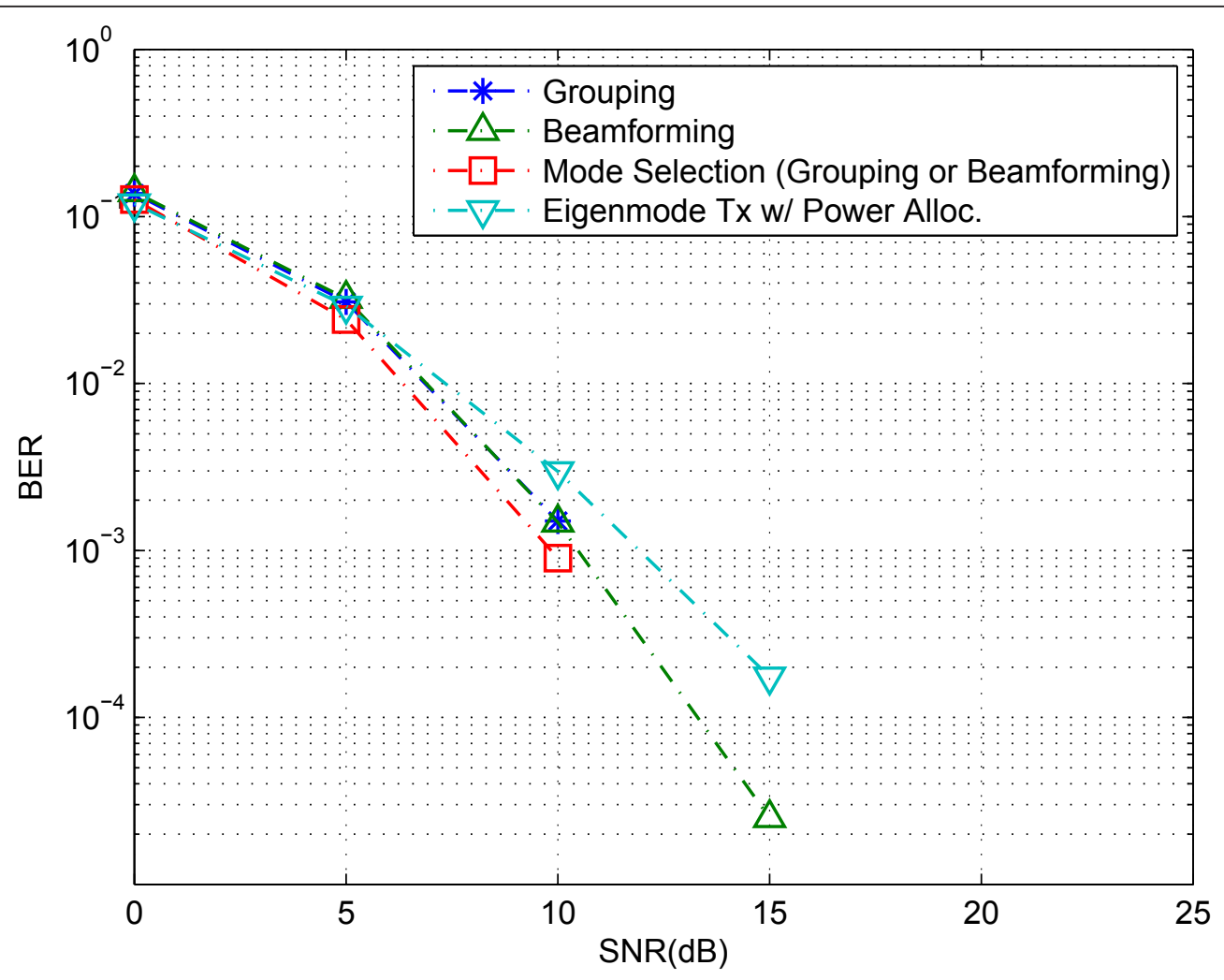

Figure 3 Average BER performance for a $4 \times 2$ system (A2 \& M1) in an IID channel.

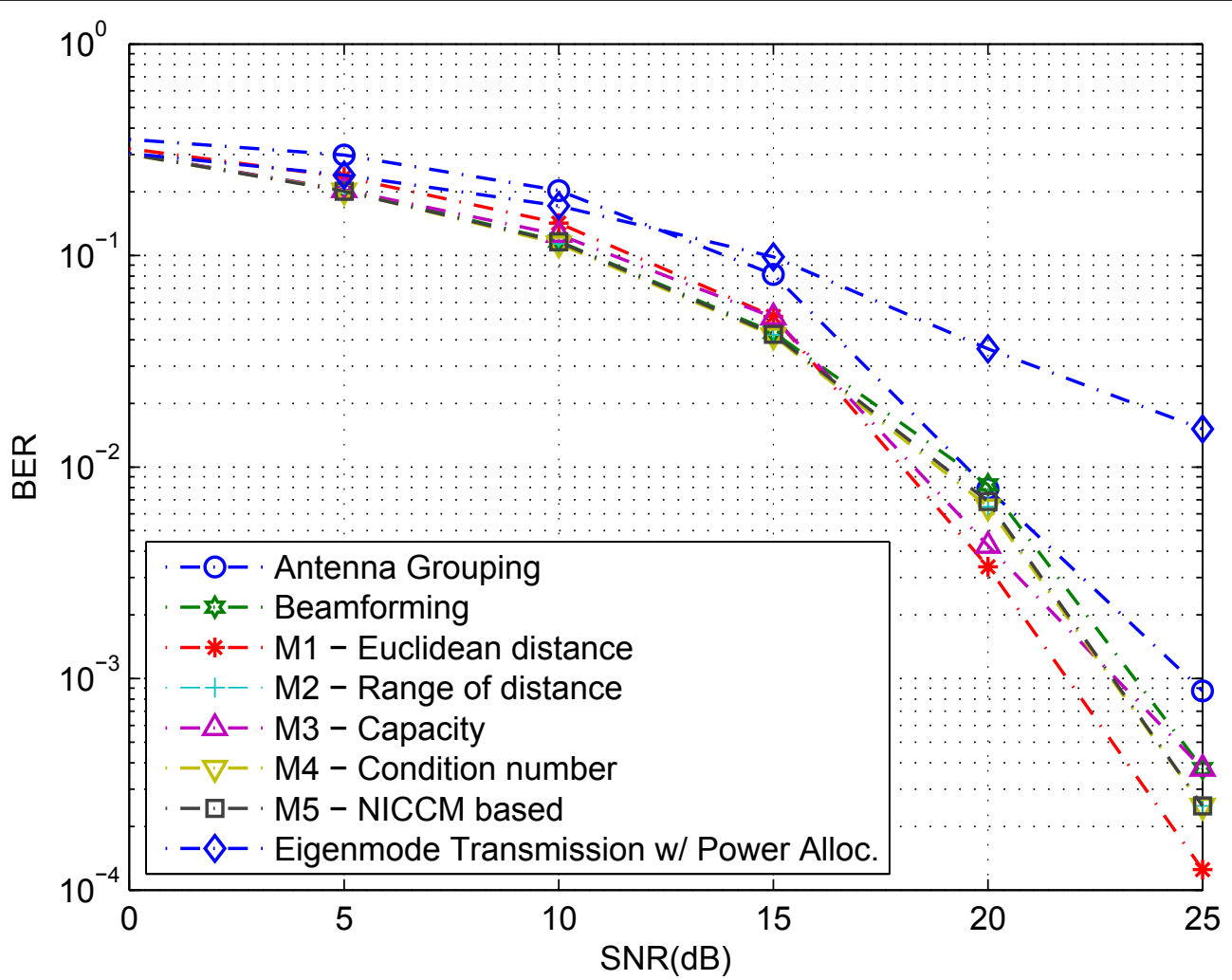

Figure 4 Comparison of mode selection algorithms combined with A2 in a correlated channel in terms of BER. 


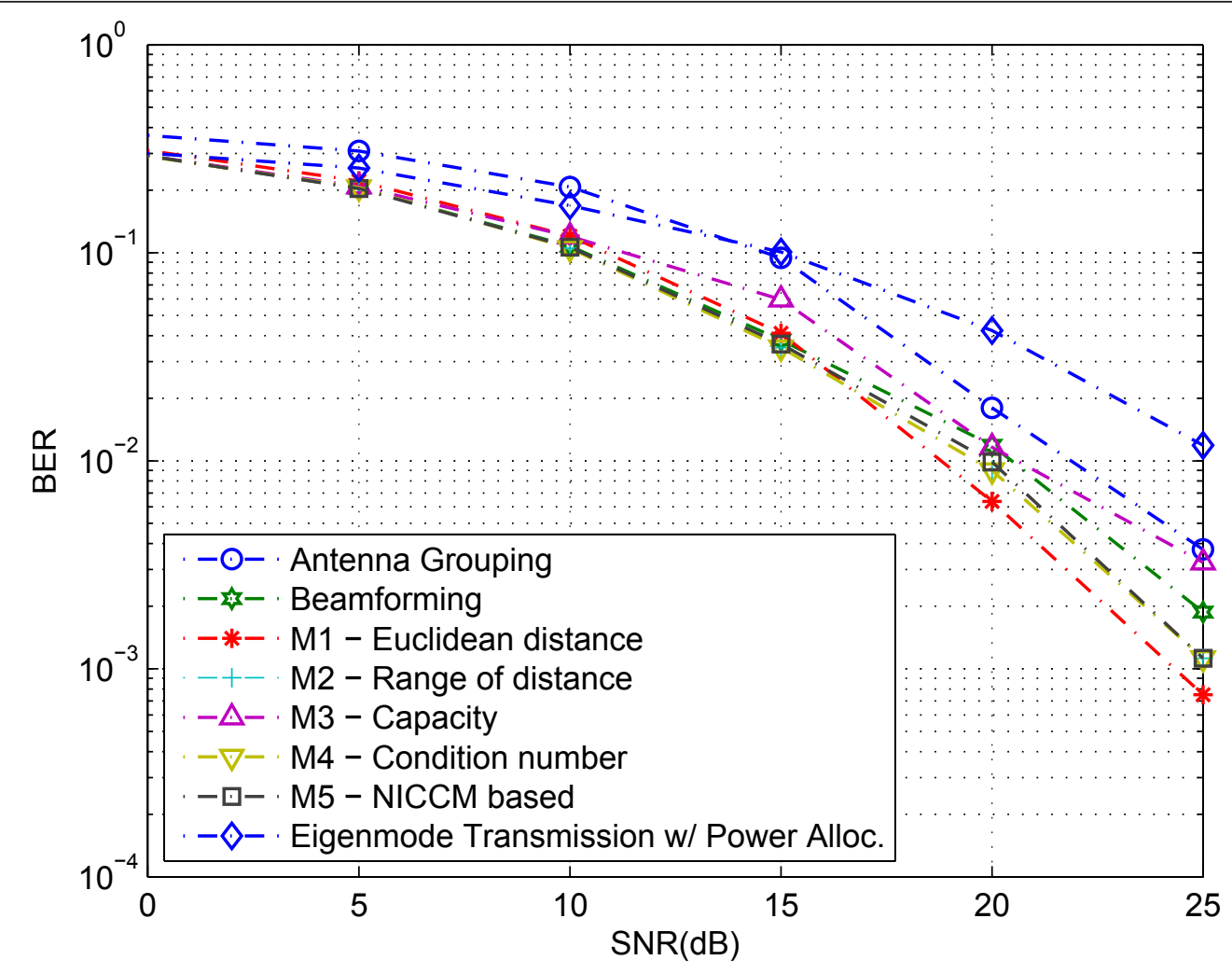

Figure 5 Comparison of mode selection algorithms combined with A5 in a correlated channel in terms of BER.

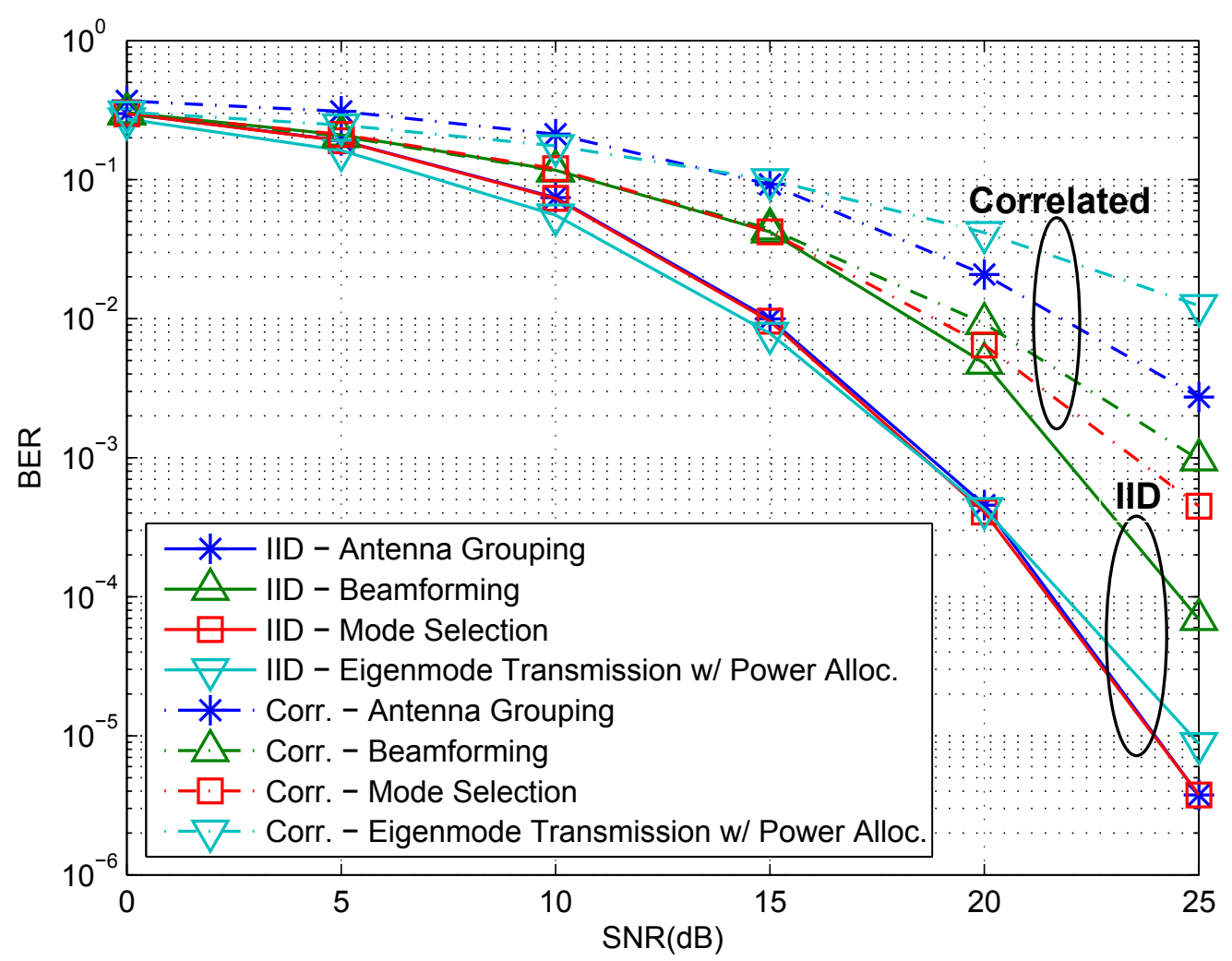

Figure 6 Average BER performance of A5 \& M5 in an IID and a correlated channel. 
threshold for the M5 algorithm. Note that the M5 algorithm does not need extra computation when it is used with the A5 algorithm which is a simple antenna grouping algorithm. It has lower complexity than other methods, and the performance of the M5 algorithm comparable to the others.

\section{Conclusions}

In an MIMO system with more transmit antennas than receive antennas, we can improve the BER performance by antenna grouping which is a hybrid form of transmit beamforming and spatial multiplexing. But antenna grouping is not always the best strategy. Using mode selection techniques, we can get robust performance irrespective of channel variation. We proposed mode selection techniques between transmit beamforming and antenna grouping for a given channel. If the channel is not ill conditioned, then we can get multiplexing gain by selecting the antenna grouping mode. When the channel is ill conditioned, we can prevent BER degradation by selecting the transmit beamforming mode. In this article, we introduce several mode selection criteria which are similar to the criteria of antenna grouping, and propose a low complexity mode selection criterion. Simulation results show that the proposed mode selection algorithm performs better than the antenna grouping and the transmit beamforming methods in various channel conditions.

\section{Endnote}

${ }^{a}$ In this article, the beamforming mode refers to the transmit beamforming mode.

\section{Acknowledgements}

This research was supported in part by the Basic Science Research Program (KRF-2008-314-D00287, 2010-0013397), the Mid-career Researcher Program (2010-0027155) through the NRF funded by the MEST, Seoul R\&BD Program (JP091007, 0423-20090051), the INMAC, and the BK21.

\section{Competing interests}

The authors declare that they have no competing interests.

Received: 27 November 2010 Accepted: 2 November 2011

Published: 2 November 2011

\section{References}

1. IE Telatar, Capacity of multi-antenna Gaussian channels. Eur Trans Telecommun. 10(6), 585-595 (1999). doi:10.1002/ett.4460100604

2. GJ Foschini, MJ Gans, On limits of wireless communication a fading environment when using multiple antennas. Wirel Personal Commun. 6(3), 311-335 (1998). doi:10.1023/A:1008889222784

3. A Goldsmith, SA Jafar, N Jindal, S Vishwanath, Capacity limits of MIMO channels. IEEE J Sel Areas Commun. 21(5), 684-702 (2003). doi:10.1109/ JSAC.2003.810294

4. H Sampath, AJ Paulraj, Joint transmit and receive optimization for high data rate wireless communication using multiple antennas, in 33rd IEEE Asilomar Conference on Signals, Systems, and Computers, vol. 1. Pacific Grove, CA, 215-219 (October 1999)

5. DJ Love, RW Heath, Multimode precoding for MIMO wireless systems. IEEE Trans Signal Process. 53(10, part 1), 3674-3687 (2005)
6. RW Heath, DJ Love, Multimode antenna selection for spatial multiplexing systems with linear receivers. IEEE Trans Signal Process. 53(8), 3042-3056 (2005)

7. N Khaled, C Desset, S Thoen, HD Man, Spatial-mode selection for the joint transmit and receive MMSE design. EURASIP J Appl Signal Process. 2004(9), 1199-1211 (2004)

8. I Kim, K Lee, J Chun, A MIMO antenna structure that combines transmit beamforming and spatial multiplexing. IEEE Trans Wirel Commun. 6(3), 775-779 (2007)

9. K Kim, J Lee, Antenna grouping technique for MIMO beamforming systems, in Proceedings of IEEE Asilomar Conference on Signals, Systems, and Computers (October, 2008)

10. R Heath, A Paulraj, Antenna selection for spatial multiplexing systems based on minimum error rate, in IEEE ICC'01. 7, 2276-2280 (2001)

11. R Heath, A Paulraj, Switching between diversity and multiplexing in MIMO systems. IEEE Trans Commun. 53(6), 962-968 (2005). doi:10.1109/ TCOMM.2005.849774

doi:10.1186/1687-1499-2011-154

Cite this article as: Kim et al:: Adaptive selection of antenna grouping and beamforming for MIMO systems. EURASIP Journal on Wireless Communications and Networking 2011 2011:154.

\section{Submit your manuscript to a SpringerOpen ${ }^{\mathcal{O}}$ journal and benefit from:}

- Convenient online submission

- Rigorous peer review

- Immediate publication on acceptance

- Open access: articles freely available online

- High visibility within the field

Retaining the copyright to your article

Submit your next manuscript at $>$ springeropen.com 